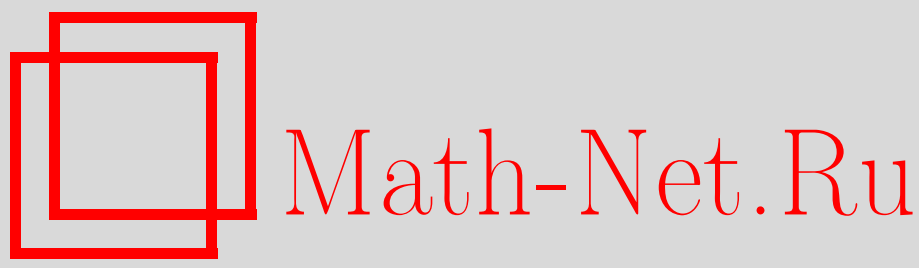

С. М. Дудаков, Трансляционная теорема для теорий $I$-сводимых алгебраических систем, Изв. РАН. Сер. матем., 2004, том 68, выпуск 5, 67-90

DOI: https://doi.org/10.4213/im503

Использование Общероссийского математического портала Math-Net.Ru подразумевает, что вы прочитали и согласны с пользовательским соглашением

http://www . mathnet.ru/rus/agreement

Параметры загрузки:

IP: 54.224 .60 .19

26 апреля 2023 г., 04:27:58 
УДК 510.652

\author{
С. М. Дудаков
}

\title{
Трансляционная теорема для теорий I-сводимых алгебраических систем
}

\begin{abstract}
Исследуются $I$-сводимые алгебраические системы, а также теории $I$-сводимых систем. Показано, что отсутствие независимой формулы в теории не является необходимым условием для $I$-сводимости её моделей даже для расширений арифметики Пресбургера. В частности, существует целый класс теорий - расширений арифметики Пресбургера, в которых имеется независимая формула и которые имеют $I$-сводимые модели. Показано, что из $I$-сводимости малой алгебраической системы автоматически следует, что каждая формула эквивалентна в ней некоторой $P$-ограниченной формуле, следовательно, для теорий таких систем выполнена трансляционная теорема.
\end{abstract}

\section{§1. Введение}

В настоящей работе мы исследуем $I$-сводимые алгебраические системы, которые были введены в $[1]^{1}$, а также теории $I$-сводимых систем. В работе [1] было показано, что каждая теория, в которой отсутствует независимая формула, имеет I-сводимые модели. Основной целью работ [7] и [9] было доказательство того, что для определенного класса теорий (имеюших $I$-сводимые модели, в которых каждая формула эквивалентна $P$-ограниченной формуле) выполняется трансляционная теорема (см. [2], [3]), т. е. любой локально генерический относительно конечных состояний запрос в расширенной сигнатуре эквивалентен некоторому запросу в ограниченной сигнатуре.

Однако для доказательства трансляционной теоремы в [7] и [9] использовано не только свойство $I$-сводимости моделей, но и еше одно условие: каждая формула должна быть эквивалентна $P$-ограниченной формуле. В работе [1] показано, что существование таких моделей следует из отсутствия в теории независимой формулы (см. [6]). Мы показываем, что отсутствие независимой формулы в теории не является необходимым условием для $I$-сводимости ее моделей даже для расширений арифметики Пресбургера. В частности, существует цельй класс теорий расширений арифметики Пресбургера, в которых имеется независимая формула и которые имеют $I$-сводимые модели.

Основная задача настоящей работы - доказательство того, что из $I$-сводимости малой алгебраической системы следует, что каждая формула эквивалентна в ней

\footnotetext{
Работа выполнена при финансовой поддержке РФФИ (проект № 04-01-00015).

${ }^{1}$ В работе [1] это свойство называется $P$-сводимостью и используется как техническое средство для доказательства трансляционной теоремы в теориях без независимой формулы; I является неразличимой ( $а н г л$. Indiscernible) последовательностью в алгебраической системе, а $P$ - именем предиката, который выделяет эту последовательность.
} 
некоторой $P$-ограниченной формуле, значит, для теорий таких систем вьполнена транслящионная теорема. В $\S 2$ мы даем основные определения, в $\S 3$ приводим примеры теорий с независимой формулой, которые имеют $I$-сводимые модели, доказывая, таким образом, что наш результат превосходит результат работы [1]. Поскольку построение примера и доказательство его корректности являются достаточно громоздкими, для удобства изложения $\S 3$ разбит на пункты. Все определения, которые используются в $\S 3$ и нигде далее, даны в п. 3.1.

В $\S 4$ мы исследуем общие свойства малых $I$-сводимых систем, в $\S 5$ вводим вспомогательные технические конструкции и устанавливаем их свойства, а в $\S 6$ доказываем основные теоремы.

\section{§2. Основные определения}

Основные понятия теории моделей, которые используются в статье, изложены в работе [5]. Пусть $\Sigma$ - произвольная сигнатура, а $P$ - одноместный предикатный символ, не входящий в $\Sigma$. Далее мы не будем специально об этом упоминать.

Для определения $I$-сводимости необходимо понятие $P$-ограниченной формулы.

ОПРЕДЕЛЕНИЕ 1 [1]. Формула первого порядка $\varphi$ сигнатуры $(\Sigma, P)$ называется $P$-ограниченной, если она или не содержит символ $P$ (т.е. является формулой в сигнатуре $\Sigma$ ), или построена из $P$-ограниченных формул с помощью булевых связок и $P$-ограниченных кванторов.

Теперь можно определить свойство $I$-сводимости.

ОПРЕДЕЛЕНИЕ 2 [1]. Пусть $I$ - плотно упорядоченное множество без концевых элементов в системе $\mathfrak{A}$ сигнатуры $\Sigma$, которое является неразличимой в $\mathfrak{A}$ последовательностью. Система $\mathfrak{A}$ обладает свойством I-сводимости, если для любой $P$-ограниченной формулы $\varphi(\bar{x}, \bar{y})$ существует бескванторная порядковая формула $\psi_{\varphi}(\bar{z}, \bar{y})$ такая, что для любого набора $\bar{m} \in A$ сушествует набор $\bar{c}_{\varphi}(\bar{m}) \in I$ такой, что

$$
(\mathfrak{A}, I) \models(\forall \bar{y} \in P)\left(\varphi(\bar{m}, \bar{y}) \leftrightarrow \psi_{\varphi}\left(\bar{c}_{\varphi}(\bar{m}), \bar{y}\right)\right)
$$

$I$-сводимость будем называть эффективной, если формула $\psi_{\varphi}$ строится по формуле $\varphi$ эффективно.

Как доказано в работе [7] (см. также [1]), для I-сводимости системы достаточно, чтобы условие $I$-сводимости выполнялось лишш для формул сигнатуры $\Sigma$.

ОПРЕДЕЛЕНИЕ 3 [1]. Пусть $\mathfrak{A}-I$-сводимая алгебраическая система сигнатуры $\Sigma$ с носителем $A$. Система $\mathfrak{A}$ называется малой, если $\mathfrak{A}$ является $|I|^{+}$-насышенной, а множество $I$ является плотным полным порядком ${ }^{2}$.

В работе [1] доказано, что для всякой системы $\mathfrak{A}$ и неразличимой последовательности $I \subseteq A$ таких, что $\mathfrak{A}$ является $I$-сводимой, сушествуют система $\mathfrak{A}^{\prime} \equiv \mathfrak{A}$ и неразличимая последовательность $I^{\prime} \subseteq A^{\prime}$, имеющая такой же тип в $\mathfrak{A}^{\prime}$, как и $I$ в $\mathfrak{A}$, такие, что система $\mathfrak{A}^{\prime}$ является малой $I^{\prime}$-сводимой.

\footnotetext{
2 Эта терминология, введенная в [1], представляется не совсем удачной, так как по смыслу определения "малым" является множество $I$, а система $\mathfrak{A}$ является "большой".
} 
ОПРЕДЕЛЕНИЕ 4 [6]. Формула $\varphi(\bar{x}, \bar{y})$ называется независимой в теории $T$, если в теории $T$ для любого натурального числа $N$ сушествуют наборы $\bar{a}_{1}, \ldots, \bar{a}_{N}$ длины $|\bar{x}|$ и для любого $K \subseteq\{1, \ldots, N\}$ существует набор $\bar{b}_{K}$ длины $|\bar{y}|$ такой, что для любого $i=1, \ldots, N$

$$
i \in K \Leftrightarrow \varphi\left(\bar{a}_{i}, \bar{b}_{K}\right)
$$

Неформально это означает, что можно найти сколь угодно большие конечные множества наборов, из которых формула способна выделить произвольное подмножество. В работе [1] показано, что из отсутствия в теории независимой формулы следует $I$-сводимость некоторых ее моделей.

ОПРЕДЕЛЕНИЕ 5 [1], [7], [9]. Пусть $I$ - плотно упорядоченное множество без конщевых элементов в системе $\mathfrak{A}$ сигнатуры $\Sigma$, являющееся неразличимой в $\mathfrak{A}$ последовательностью. Алгебраическая система $\mathfrak{A}$ называется тотально I-сводимой, если для любой формулы $\varphi(\bar{x}, \bar{y})$ сигнатуры $(\Sigma, P)$ (не обязательно $P$-ограниченной) существует бескванторная порядковая формула $\psi_{\varphi}(\bar{z}, \bar{y})$ такая, что для любого $\bar{m} \in A$ существует набор $\bar{c}_{\varphi}(\bar{m}) \in I$ такой, что

$$
(\mathfrak{A}, I) \models(\forall \bar{y} \in P)\left(\varphi(\bar{m}, \bar{y}) \leftrightarrow \psi_{\varphi}\left(\bar{c}_{\varphi}(\bar{m}), \bar{y}\right)\right) .
$$

В работе [1] показано, что тотальная $I$-сводимость некоторой модели теории (как и $I$-сводимость) является следствием отсутствия в этой теории независимой формулы.

Пусть $T$ - теория сигнатуры $\Sigma$. Пусть $\Xi$ - конечная предикатная сигнатура, не имеющая общих символов с $\Sigma$, за исключением порядка $\leqslant$. При рассмотрении моделей сигнатуры $\Sigma \cup \Xi$ мы всегда считаем, что отношения порядка $\Sigma$ и $\Xi$ согласованы, следовательно, противоречий не возникает.

ОПРЕДЕЛЕНИЕ 6 [2], [3]. Запрос (формула первого порядка) в сигнатуре $\Sigma \cup \Xi$ назьвается расширенным, в сигнатуре $\Xi$ - ограниченныл. Если $\mathfrak{A}$ - модель $T$, $\mathfrak{B}$ - интерпретация символов из $\Xi$ над $\mathfrak{A}$, то $\mathfrak{B}$ называется состоянием в $\mathfrak{A}$. Состояние $\mathfrak{B}$ называется конечнылм, если интерпретации всех символов из $\Xi$ конечны. Запрос называется локально генерическим относительно конечных состояний (или просто локально генерическим), если из истинности запроса в $(\mathfrak{A}, \mathfrak{B})$ следует его истинность в $(\mathfrak{A}, \mathfrak{C})$ для любого изоморфного $\mathfrak{B}$ состояния $\mathfrak{C}$. Если каждый локально генерический расширенный запрос эквивалентен на конечных состояниях некоторому ограниченному запросу, то это свойство теории называется трансляционным результатом ${ }^{3}$ (в теории выполняется трансляционная теорема).

В работах $[7]$ и [9] доказано, что из тотальной $I$-сводимости алгебраической системы следует трансляционная теорема для теории этой системы. Основной результат настояшей работы - доказательство того, что каждая малая $I$-сводимая алгебраическая система является тотально $I$-сводимой. Таким образом, мы показываем, что $I$-сводимость какой-либо модели теории является достаточным условием для трансляционного результата.

\footnotetext{
${ }^{3}$ Aнгл. The collapse result.
} 


\section{§3. Примеры теорий с независимой формулой, которые имеют $I$-сводимые модели}

В этом параграфе мы покажем, что существуют теории, которые имеют $I$-сводимые модели, и, вместе с тем, в этих теориях существует независимая формула.

3.1. Определения. В арифметике Пресбургера выразимо умножение на любую рациональную константу (с дальнейшим округлением). Далее всюду строчные греческие буквы $\alpha, \beta$ (возможно, с индексами) будут обозначать фиксированные рациональные числа.

Мы рассматриваем модели теории $\mathrm{Th}(\omega,+)$, т. е. теории натуральных чисел со сложением. Заметим, что в теории определимы порядок:

$$
x \leqslant y \Leftrightarrow(\exists u)(x+u=y),
$$

и все натуральные числа:

$$
\begin{aligned}
& x=0 \Leftrightarrow(\forall y)(y \geqslant x), \\
& x=1 \Leftrightarrow(\forall y)(y<x \rightarrow y=0), \\
& x=n \Leftrightarrow x=\underbrace{1+\cdots+1}_{n} .
\end{aligned}
$$

Рассмотрим элементарные расширения системы $(\omega,+)$. Напомним (см., например, [4]), что всякое элементарное расширение $(\omega,+)$ состоит из начального фрагмента, который изоморфен $(\omega,+)$, и множества классов, каждый из которых порядково изоморфен множеству всех целых чисел. Эти классы образуют плотный линейный порядок, в котором начальный фрагмент является наименьшим элеменTOM.

Рассмотрим произвольную нестандартную модель $\mathfrak{A}=\left(A,+^{\mathfrak{A}}\right)$ теории $\operatorname{Th}(\omega,+)$. Пусть $P$ - одноместный предикатный символ и $P^{\mathfrak{A}}$ - его интерпретация такая, что $P^{\mathfrak{A}} \subseteq \omega$. Предположим, что предикат $P^{\mathfrak{A}}$ неограничен на $\omega$, т. е. выполняется

$$
(\forall x)(\exists y>x)(P(x) \rightarrow P(y)) \wedge(\exists x) P(x) .
$$

Тогда в теории $\operatorname{Th}\left(\mathfrak{A}, P^{\mathfrak{A}}\right)$ определимо множество натуральных чисел $\omega$ :

$$
x \in \omega \Leftrightarrow(\exists y \geqslant x) P(y) .
$$

Определим отношение $\cong$ следуюшим образом:

$$
x \cong y \Leftrightarrow(\exists z \in \omega)(x+z=y \vee y+z=x) .
$$

Неформально это означает, что $x$ и $y$ принадлежат одному классу.

Введем отношения:

$$
\begin{aligned}
& x \ll y \Leftrightarrow x<y \wedge \neg(x \cong y), \\
& x \lll y \Leftrightarrow x<y \vee x \cong y .
\end{aligned}
$$

Первое означает, что $x$ лежит в меньшем чем $y$ классе, а второе - в меньшем или в том же самом. 
Очевидно, что символы $\omega$ и $\cong$ которые мы определили, выражаются через отношения «и $\ll$ :

$$
\begin{gathered}
x \in \omega \Leftrightarrow x \lll 0, \\
x \cong y \Leftrightarrow x \lll y \wedge y \lll x .
\end{gathered}
$$

Отрицание перед отношениями « и $ґ$ убирается точно так же, как для плотного линейного порядка:

$$
\begin{aligned}
& \neg(x \ll y) \Leftrightarrow y \ll x, \\
& \neg(x \ll y) \Leftrightarrow y \ll x .
\end{aligned}
$$

Будем использовать также вычитание, когда оно имеет смысл для натуральных чисел, и умножение на фиксированное рациональное число. Все эти отношения определимы, а добавление к сигнатуре определимых символов не увеличивает выразительных возможностей, поэтому далее мы будем включать все упомянутые символы в состав сигнатуры.

Пусть $I \subseteq A$. Пусть $\mathcal{T}$ - множество всевозможных термов сигнатуры $\Sigma$ с одной свободной переменной $x$. Множество $I$ называется термально изолированным в $\mathfrak{A}$ (см. [8]), если для любых термов $t_{1}(x), t_{2}(x) \in \mathcal{T}$ имеет место один из двух случаев:

1) сушествует константа $M_{t_{2}} \in \omega$ такая, что для всех $a \in I$ выполнено неравенство $t_{2}(a) \leqslant M_{t_{2}}$

2) для любых элементов $a, b \in I$ таких, что $a<b$, выполнено $t_{1}(a)<t_{2}(b)$.

Известно (см., например, [8]), что существуют элементарные расширения $(\omega,+)$, в которых есть термально изолированные неразличимые множества, и если $(\mathfrak{A}, I)$ - такая система и $(\mathfrak{B}, J) \equiv(\mathfrak{A}, I)$, то $J$ будет термально изолированным неразличимым в $\mathfrak{B}$.

Класс изоляиии элемента $a$ - это множество $[a]$ :

$$
[a]=\bigcup\left\{\left[t_{1}(a), t_{2}(a)\right]: t_{1}, t_{2} \in \mathcal{T} \wedge t_{1}(a) \notin \omega\right\},
$$

где $[x, y]$ - обычный отрезок. Если множество $I \subseteq A$ термально изолировано, то для всех $x, y \in I$ таких, что $x \neq y$, классы изоляции $[x]$ и $[y]$ не пересекаются.

3.2. Свойства предикатных обогащений начального фрагмента нестандартных моделей. Пусть теория $T=\operatorname{Th}\left(\omega,+,\left(P_{i}\right)_{i}\right)$, где $P_{i}$ - произвольные предикаты, допускает элиминацию кванторов. Рассмотрим $\mathfrak{A} \succ(\omega,+)$ - элементарное (строгое) расширение арифметики Пресбургера. Мы будем изучать теорию $T^{\prime}=\operatorname{Th}\left(\mathfrak{A},\left(P_{i}\right)_{i}\right)$.

Прежде всего докажем, что теория $T^{\prime}$ также допускает элиминицию кванторов.

ЛЕмма 1. Теория $T^{\prime}$ допускает әлиминацию кванторов.

ДокАЗАТЕЛЬство. Если предикат $P_{l}$ ограничен на множестве $\omega$, то

$$
P_{l}(\bar{x}) \Leftrightarrow \bigvee_{\bar{c} \in P_{l}} \bar{x}=\bar{c} .
$$

Следовательно, все такие предикаты можно выбросить, не уменьшив выразительной силы теории. 
Пусть хотя бы один предикат из $P_{l}$ неограничен на $\omega$. Рассмотрим произвольную формулу

$$
\varphi \sim(\exists x)\left(\left(t_{1} \leqslant \alpha x \leqslant t_{2}\right) \wedge\left(s_{1} \ll_{1}^{*} \beta x \ll_{2}^{*} s_{2}\right) \wedge\left(\bigwedge_{i}(\neg)^{\varepsilon_{i}} P_{j_{i}}\left(\bar{r}_{i}\right)\right)\right) .
$$

Здесь $\varepsilon_{i} \in\{0 ; 1\},(\neg)^{\varepsilon}$ означает $\neg$, если $\varepsilon=1$, или пустое слово, если $\varepsilon=0$. С помошью $\ll_{k}^{*}$ здесь и далее обозначается одно из отношений $\ll$ или $\ll$.

Достаточно рассматривать только формулы такого вида, потому что если имеется несколько неравенств, например

$$
t_{11} \leqslant \alpha_{i} x \wedge \cdots \wedge t_{1 n} \leqslant \alpha_{n} x
$$

то можно рассмотреть $n$ случаев вида " $t_{1 i_{0}} / \alpha_{i_{0}}-$ наибольший из $t_{1 i} / \alpha_{i}$ ", взять дизъюнкцию по всем этим случаям, и тогда в каждом отдельном случае указанная конъюнкция будет эквивалентна $t_{1 i_{0}} \leqslant \alpha_{i_{0}} x$ (если все $\alpha_{i}$ положительны).

Если вхождений $P_{j_{i}}\left(\bar{r}_{i}\right)$ нет, то квантор удаляется аналогично арифметике Пресбургера.

Для любого $y$ выполнено

$$
y \lll 0 \vee y \gg 0 .
$$

Также очевидна эквивалентность

$$
\left(\bigvee_{k} y^{(k)} \gg 0\right) \wedge \neg P_{i}(\bar{y}) \Leftrightarrow \bigvee_{k} y^{(k)} \gg 0
$$

и то, что следуюшая формула является тождественно ложной:

$$
\left(\bigvee_{k} y^{(k)} \gg 0\right) \wedge P_{i}(\bar{y})
$$

Здесь $y^{(k)}-k$-я компонента набора $\bar{y}$. Следовательно, можно считать, что если формула $\varphi$ содержит $P_{j_{i}}\left(\bar{r}_{i}\right)$, то для всех $r_{i}^{(k)}$ выполнено $r_{i}^{(k)} \lll 0$. Если это не так, то все сводится к случаю с меньшим количеством вхождений $P_{j_{i}}$. Выберем произвольное $k$ такое, что

$$
r_{i}^{(k)} \sim p_{i}^{(k)}+\alpha_{i}^{(k)} x,
$$

где $p_{i}^{(k)}$ не содержит $x$, и обозначим

$$
y=p_{i}^{(k)}+\alpha_{i}^{(k)} x .
$$

Тогда, заменив всюду $x$ на $\left(y-p_{i}^{(k)}\right) / \alpha_{i}^{(k)}$, вместо $\varphi$ мы получим формулу $\varphi^{\prime}$ :

$$
(\exists y \in \omega)\left(\left(t_{1}^{\prime} \leqslant \alpha^{\prime} y \leqslant t_{2}^{\prime}\right) \wedge\left(s_{1}^{\prime} \ll_{1}^{*} \beta^{\prime} y \ll_{2}^{*} s_{2}^{\prime}\right) \wedge\left(\bigwedge_{i}(\neg)^{\varepsilon_{i}} P_{j_{i}}\left(\bar{r}_{i}^{\prime}\right)\right)\right)
$$

где каждый $r_{i}^{(k)}$ имеет вид ${p^{\prime}}_{i}^{(k)}+{\alpha^{\prime}}_{i}^{(k)} y$. Упростим её.

Если $t_{1}^{\prime} \gg 0$, то формула ложна. Если $t_{2}^{\prime} \gg 0$, то неравенство $\alpha^{\prime} y \leqslant t_{2}^{\prime}$ является истинным и его можно исключить из формулы, не изменив ее истинности. Таким образом, можно считать, что $t_{1}^{\prime}$ и $t_{2}^{\prime}$ - натуральные числа. 
Поскольку $y \in \omega$, то $\beta^{\prime} y \in \omega$ и двойное неравенство

$$
s_{1}^{\prime} \ll_{1}^{*} \beta^{\prime} y \ll_{2}^{*} s_{2}^{\prime}
$$

сводится к неравенству

$$
s_{1}^{\prime} \ll_{1}^{*} 0 \ll_{2}^{*} s_{2}^{\prime},
$$

которое не содержит $y$ и выносится из-под квантора.

Так как для всех ${r^{\prime}}_{i}^{(k)}$ выполнено ${r^{\prime}}_{i}^{(k)} \ll 0$ и $y \in \omega$, то и ${p^{\prime}}_{i}^{(k)} \in \omega$.

Итак, мы выяснили, что можно ограничиться только случаем, когда:

1) $t_{1}^{\prime}, t_{2}^{\prime} \in \omega$

2) неравенства «и цлля $у$ отсутствуют;

3) ${p^{\prime}}_{i}^{(k)} \in \omega$ для всех $i, k$.

Однако эта ситуация означает, что мы находимся внутри модели $\left(\omega,+,\left(P_{i}\right)_{i}\right)$ и формула $\varphi^{\prime}$ эквивалентна бескванторной по свойству исходной теории $T$.

СлеДСТВИЕ 1. Если теория Т допускает әффективную әлиминачию кванторов, то әлиминация кванторов в теории $T^{\prime}$ тожсе выполняется эффективно.

ЛЕмма 2. Пусть $\mathfrak{B}$ - произвольная модель $T^{\prime}$. Пусть $I$ - произвольное термально изолированное множество в $\mathfrak{B}$, все әлементы которого больше $\omega^{\mathfrak{B}}\left(\omega^{\mathfrak{B}}\right.$ - интерпретация символа $\omega$ в $\mathfrak{B}-$ может не совпадать с множеством натуральных чисел). Если

$$
\sum_{i=1}^{k} \alpha_{i} d_{i} \in \omega^{\mathfrak{B}}
$$

для каких-либо попарно различных $d_{i}$, то $\alpha_{i}=0$ для $i=1, \ldots, k$.

ДоказАТЕЛЬСтво. Предположим, что утверждение не верно, и для некоторых $\alpha_{i}$ (не равных нулю), $i=1, \ldots, k$, имеем

$$
\sum_{i=1}^{k} \alpha_{i} d_{i} \in \omega^{\mathfrak{B}} .
$$

Выберем $d_{i_{0}}-$ самое большое из $d_{i}$. Если $k=1$, то

$$
\alpha_{1} d_{1} \in \omega^{\mathfrak{B}}
$$

и, следовательно, $d_{1} \in \omega^{\mathfrak{B}}$, что противоречит тому, что все элементы $I$ не входят В $\omega^{\mathfrak{B}}$.

Пусть $k \geqslant 2$. Если $d_{i_{1}}-$ второе по величине среди $d_{i}$, то по свойству термальной изолированности получим

$$
d_{i_{0}} \gg \gamma d_{i_{1}}
$$

для любого натурального $\gamma$. Пусть

$$
\gamma \geqslant \frac{\sum_{i=1}^{k}\left|\alpha_{i}\right|}{\left|\alpha_{i_{0}}\right|}
$$


Тогда

$$
d_{i_{0}} \gg \gamma d_{i_{1}} \geqslant \frac{\sum_{i=1}^{k}\left|\alpha_{i}\right|}{\left|\alpha_{i_{0}}\right|} d_{i_{1}} \geqslant \frac{\sum_{i \neq i_{0}}\left|\alpha_{i}\right| d_{i}}{\left|\alpha_{i_{0}}\right|} .
$$

Следовательно, $\left|\alpha_{i_{0}}\right| d_{i_{0}} \gg \sum_{i \neq i_{0}}\left|\alpha_{i}\right| d_{i}$. Получаем

$$
\left|\sum_{i=1}^{k} \alpha_{i} d_{i}\right| \geqslant\left|\alpha_{i_{0}}\right| d_{i_{0}}-\sum_{i \neq i_{0}}\left|\alpha_{i}\right| d_{i} \gg 0,
$$

что противоречит предположению.

СЛЕДСТвиЕ 2. Если

$$
\sum_{i=1}^{k} \alpha_{i} d_{i} \cong \sum_{i=1}^{k} \alpha_{i} d_{i}^{\prime}
$$

для каких-либо $d_{i}, d_{i}^{\prime} \in I$, причем $d_{i}$ и $d_{i}^{\prime}$ упорядочены по убьванию:

$$
\begin{aligned}
& d_{1}>d_{2}>\cdots>d_{k}, \\
& d_{1}^{\prime}>d_{2}^{\prime}>\cdots>d_{k}^{\prime},
\end{aligned}
$$

$\alpha_{i} \neq 0$ для $i=1, \ldots, k$, mо $_{i}=d_{i}^{\prime}$ для всех $i$.

ДокАЗАТЕЛЬСТво. Если это не так, то

$$
\sum_{i=1}^{k} \alpha_{i}\left(d_{i}-d_{i}^{\prime}\right) \in \omega^{\mathfrak{B}} .
$$

Дальнейшие рассуждения аналогичны доказательству леммы 2.

3.3. $I$-сводимость. Напомним, что предикаты $P_{i}$ определены на множестве $\omega$ так, что теория $T=\operatorname{Th}\left(\omega,+,\left(P_{i}\right)_{i}\right)$ допускает элиминацию кванторов и $\mathfrak{A} \succ$ $(\omega,+)$. Теперь можно доказать, что некоторые модели теории $T^{\prime}=\operatorname{Th}\left(\mathfrak{A},\left(P_{i}\right)_{i}\right)$ являются $I$-сводимыми для подходяшего $I$.

ТеОрема 1. Пусть $\mathfrak{B}$ - произвольная модель $T^{\prime}$ с носителем В. Пусть $I \subseteq B$ - произвольное термально изолированное неразличимое множество в $\mathfrak{B}$, все әлементы которого больие $\omega^{\mathfrak{B}}$. Пусть система $\mathfrak{B}$ является малой. Тогда система $\left(\mathfrak{B},\left(P_{i}\right)_{i}\right)$ является $I$-сводимой.

ДокАЗАтЕЛЬСтво. Рассмотрим произвольную формулу $\varphi(\bar{x}, \bar{y})$. Необходимо доказать, что сушествует порядковая формула $\psi_{\varphi}$ такая, что для всякого $\bar{m} \in B$ сушествуют такие $\bar{c}_{\varphi}(\bar{m}) \in I$, что

$$
(\mathfrak{B}, I) \models(\forall \bar{d} \in I)\left(\varphi(\bar{m}, \bar{d}) \leftrightarrow \psi_{\varphi}\left(\bar{c}_{\varphi}(\bar{m}), \bar{d}\right)\right) .
$$

Согласно лемме 1 можно считать, что формула $\varphi$ бескванторная. Покажем, что формула $\psi_{\varphi}$ сушествует для каждой атомной формулы $\varphi$, откуда будет следовать утверждение в обшем случае.

Атомная формула может иметь один из трех видов:

$$
P_{i}\left(\sum \alpha_{i} d_{i}+\sum \beta_{i} m_{i}\right), \quad \sum \alpha_{i} d_{i} \ll^{*} \sum \beta_{i} m_{i}, \quad \sum \alpha_{i} d_{i}<^{*} \sum \beta_{i} m_{i} .
$$


Здесь мы полагаем, что $\bar{d}$ - попарно различные элементы $I$, упорядоченные по убыванию, а $\bar{m}$ - произвольные элементы $B$. Мы будем рассматривать только строгие неравенства, нестрогие рассматриваются аналогично.

Для каждого терма $t$, который входит в $P_{i}(\bar{t})$, добавим дизъюнкцию

$$
t \ll 0 \vee 0 \ll t
$$

и раскроем скобки. Как и ранее, если $0 \ll t$ для некоторого терма $t$ из $\bar{t}$, то формула $P_{i}(\bar{t})$ ложна, поэтому можно считать, что для всех термов $t$, которые входят в формулы вида $P_{i}(\bar{t})$, выполнено $t \cong 0$.

Согласно следствию 2 для каждого терма вида

$$
t \sim \sum \alpha_{i} d_{i}+\sum \alpha_{i} m_{i}
$$

сушествует не более одного набора $\bar{d} \in I$, для которого $t(\bar{d}) \cong 0$. Следовательно, на множестве $I$ формула $t \cong 0$ или ложна для любых $\bar{d}$, или эквивалентна формуле $\bar{d}=\bar{d}^{*}$ для некоторого набора $\bar{d}^{*}$.

Если формула $\bar{t} \cong \overline{0}$ ложна на $I$ для любых $\bar{d}$, то любая формула $P_{i}(\bar{t})$ тоже ложна. В противном случае существует только один набор $\bar{d}^{*}$, на котором формула $\bar{t} \cong \overline{0}$ истинна. Тогда в $P_{i}(\bar{t})$ достаточно подставить значение термов $\bar{t}$ на этом наборе. Таким образом, для каждого $\bar{m} \in A$ можно найти константы $c_{1}, c_{2}, \bar{d}^{*} \in I$ такие, что для всех $\bar{d} \in I$ будет выполнено

$$
P_{i}(\bar{t}(\bar{d})) \Leftrightarrow c_{1}=c_{2} \wedge \bar{d}=\bar{d}^{*} .
$$

Второй случай:

$$
\sum \alpha_{i} d_{i} \ll \sum \beta_{i} m_{i}
$$

Это неравенство эквивалентно тому, что

$$
\left(\sum \alpha_{i} d_{i}<\sum \beta_{i} m_{i}\right) \wedge \neg\left(\sum \alpha_{i} d_{i} \cong \sum \beta_{i} m_{i}\right) .
$$

Однако формула

$$
\sum \alpha_{i} d_{i} \cong \sum \beta_{i} m_{i}
$$

или ложна на $I$ для любых $\bar{d}$, или эквивалентна $\bar{d}=\bar{d}^{*}$ для некоторого $\bar{d}^{*}$.

Третий случай:

$$
\sum \alpha_{i} d_{i}<\sum \beta_{i} m_{i}
$$

Положим

$$
M=\sum \beta_{i} m_{i}
$$

Поскольку множество $I$ термально изолировано, то существует не более одного элемента $d^{*} \in I$ такого, что $M \in\left[d^{*}\right]$.

Покажем, что всякая формула указанного выше вида, если $\bar{d}$ пробегают $I$, а $M$ фиксировано, эквивалентна порядковой формуле с константами из $I$ индукцией по количеству $d$ независимо от $M$. Если $d$ отсутствуют, то это очевидно.

Предположим, что в сумме $\sum \alpha_{i} d_{i}$ все элементы $d_{i}$ различны. В противном случае, приводя подобные члены, мы получим другую формулу с меньшим числом $d$. Будем считать, что $M>0$. Другие случаи ничем принципиально не отличаются. 
Сначала рассмотрим случай, когда $M \in\left[d^{*}\right]$ для некоторого $d^{*} \in I$.

Итак, рассмотрим неравенство вида

$$
\sum \alpha_{i} d_{i}<M
$$

1) Если $d_{1}>d^{*}$, то $\sum \alpha_{i} d_{i}>M$ при $\alpha_{1}>0$ и $\sum \alpha_{i} d_{i}<M$ при $\alpha_{1}<0$. Следовательно,

$$
\sum \alpha_{i} d_{i}<M \Leftrightarrow \alpha_{1}<0
$$

2) Если $d_{1}<d^{*}$, то неравенство $\sum \alpha_{i} d_{i}<M$ истинно.

$3)$ Если $d_{1}=d^{*}$, то, перенеся $d_{1}$ в правую часть, мы получим

$$
\sum_{i>1} \alpha_{i} d_{i}<M-\alpha_{1} d^{*}
$$

По индукционному предположению эта формула эквивалентна порядковой формуле $\psi^{\prime}\left(\bar{c}, \bar{d}^{\prime}\right)$ для некоторых констант $\bar{c}$ и для всех $\bar{d}^{\prime}$.

Тогда неравенство

$$
\sum \alpha_{i} d_{i}<M
$$

эквивалентно формуле

$$
\left(d_{1}>d^{*}\right) \wedge\left(\alpha_{1}<0\right) \vee\left(d_{1}<d^{*}\right) \vee\left(d_{1}=d^{*} \wedge \psi^{\prime}\left(\bar{c}, \bar{d}^{\prime}\right)\right) .
$$

Теперь предположим, что $M \notin[d]$ для всех $d \in I$. Возможны следуюшие варианты: $M<d$ для всех $d \in I, M>d$ для всех $d \in I$ или $d_{1}<M<d_{2}$ для некоторых $d_{1}, d_{2} \in I$. В первом случае

$$
\sum \alpha_{i} d_{i}<M \Leftrightarrow \alpha_{1}<0
$$

для любых $\bar{d}$, во втором случае $\sum \alpha_{i} d_{i}<M$ для любых $\bar{d}$.

Пусть теперь $M$ находится в "середине" $I$. Разобьем $I$ на два класса, меньших $M$ и больших $M$, элементов. Оба класса непусты, не пересекаются, их объединение равно $I$. Поскольку $I$ является плотным полным порядком, то или в меньшем классе существует наибольший элемент, или в большем существует наименьший. Рассмотрим первый случай. Пусть $d^{*}$ - наибольший элемент $I$, который меньше $M$. Тогда неравенство

$$
\sum \alpha_{i} d_{i}<M
$$

эквивалентно формуле

$$
d_{1} \leqslant d^{*} \vee\left(d_{1}>d^{*} \wedge \alpha_{1}<0\right) .
$$

Аналогично во втором случае.

СЛЕДСТВИЕ 3. Если теория Т допускает әффективную әлиминацию кванторов, то система $\left(\mathfrak{B},\left(P_{i}\right)_{i}\right)$ будет әффективно I-сводимой.

ТЕорема 2. Существуют обогащения арифметики Пресбургера, которые имеют независимую формулу и при этом имеют әффективно I-сводимые модели. 
ДокАЗАТЕЛЬСтво. Рассмотрим произвольное обогашение системы счетным числом предикатов $T=\operatorname{Th}\left(\omega,+,\left(P_{i}\right)_{i}\right)$. Пусть $T^{\prime \prime}$ - морлиевское обогашение теории $T$, т. е. для всякой формулы $\varphi(\bar{x})$ добавлен предикатный символ $R_{\varphi}$ и теория $T^{\prime \prime}$ содержит формулу

$$
(\forall \bar{x})\left(R_{\varphi}(\bar{x}) \leftrightarrow \varphi(\bar{x})\right) .
$$

Тогда $T^{\prime \prime}$ допускает эффективную элиминацию кванторов. Кроме того, $T^{\prime \prime}$ имеет модель $\left(\omega,+,\left(P_{i}\right)_{i},\left(R_{\varphi}\right)_{\varphi}\right)$. Таким образом, $T^{\prime \prime}$ удовлетворяет условиям теоремы 1. Следовательно, выбрав нестандартную модель $\mathfrak{A}$, можно построить эффективно $I$-сводимую модель для теории $\operatorname{Th}\left(\mathfrak{A},\left(P_{i}\right)_{i},\left(R_{\varphi}\right)_{\varphi}\right)$.

Пусть предикат $P$ :

$$
P(x, y, z) \Leftrightarrow x y=z,
$$

определяет умножение на натуральных числах. Тогда в теории $T=\operatorname{Th}(\omega,+, P)$ есть независимая формула. Пусть $\left(\omega,+, P,\left(R_{\varphi}\right)_{\varphi}\right)$ - морлиевское обогащение $T$. Согласно теоремам 1,2 теория $T^{\prime}=\operatorname{Th}\left(\mathfrak{A}, P,\left(R_{\varphi}\right)_{\varphi}\right)$ обладает всеми указанными в теореме свойствами. Заметим, что если в теории $T$ имеется независимая формула $\varphi$, то эта формула останется независимой и в теории $T^{\prime}$.

\section{§4. Свойства $I$-сводимых алгебраических систем}

Рассмотрим произвольную малую алгебраическую систему $\mathfrak{A}$ сигнатуры $(\Sigma, P)$, которая обладает свойством $I$-сводимости, $I$ - плотно упорядоченное множество без концевых элементов, являюшееся неразличимой в $\mathfrak{A}$ последовательностью.

Для доказательства тотальной $I$-сводимости системы $\mathfrak{A}$ покажем, что для каждой $P$-ограниченной формулы $\varphi(x, \bar{y})$ можно найти $P$-ограниченную формулу $\varphi^{\prime}(\bar{y})$ такую, что

$$
(\mathfrak{A}, I) \models(\forall \bar{y})\left((\exists x) \varphi(x, \bar{y}) \leftrightarrow \varphi^{\prime}(\bar{y})\right) .
$$

Сначала установим одно простое свойство порядковых формул.

ЛЕмма 3. Пусть $\psi(\bar{z}, \bar{y})$ - произвольная бескванторная порядковая формула. Если наборы $\bar{d}_{1}$ и $\bar{d}_{2}$ одинаково упорядочень, их әлементы, лехсащие вне отрезка $[a, b]$, совпадают (m. е. если $d_{1}^{(i)} \notin[a, b]$ или $d_{2}^{(i)} \notin[a, b]$, mо $\left.d_{1}^{(i)}=d_{2}^{(i)}\right)$ $u$

$$
\psi\left(\bar{c}, \bar{d}_{1}\right) \nleftarrow \psi\left(\bar{c}, \bar{d}_{2}\right),
$$

то на отрезке $[a, b]$ лежит хотя бы один әлемент набора $\bar{c}$.

ДокАЗАТЕльСтво. Если внутри этого отрезка нет ни одного элемента $\bar{c}$, то $\bar{d}_{1}$ и $\bar{d}_{2}$ имеют одинаковый порядковый тип над $\bar{c}$, следовательно, формулы $\psi\left(\bar{c}, \bar{d}_{1}\right)$ и $\psi\left(\bar{c}, \bar{d}_{2}\right)$ должны быть эквивалентны.

Из леммы 3 и $P$-сводимости сразу получаем

СЛЕДСТВИЕ 4. Пусть $\varphi(\bar{x}, \bar{y})$ - произвольная Р-ограниченная формула, $\bar{m} \in A, \quad a, b, \bar{d}_{1}, \bar{d}_{2} \in I, \quad a<b$. Eсли наборы $\bar{d}_{1}$ и $\bar{d}_{2}$ одинаково упорядоченьь, их әлементыл, лежсащие вне отрезка $[a, b]$, совпадают $и$

$$
(\mathfrak{A}, I) \models \varphi\left(\bar{m}, \bar{d}_{1}\right) \leftrightarrow \varphi\left(\bar{m}, \bar{d}_{2}\right),
$$

то на отрезке $[a, b]$ лежит хотя бы один әлемент набора $\bar{c}_{\varphi}(\bar{m})$. 
Всякая $P$-ограниченная формула, очевидно, эквивалентна $P$-ограниченной предваренной формуле. Однако верно более сильное утверждение: можно добиться того, чтобы в этой предваренной формуле $P$-ограниченные кванторы образовывали приставку вида $\exists \forall$.

Лемма 4 (см. также [1]). Всякая Р-ограниченная формула әквивалентна предваренной Р-ограниченной формуле, в которой $P$-ограниченные кванторы образуют приставку вида $\exists \forall$.

ДокАЗАТЕЛЬСтво. Пусть $\varphi(\bar{y}, \bar{w})$ - произвольная формула сигнатуры $\Sigma$. Тогда

$$
(\mathfrak{A}, I) \models(\forall \bar{y})(\exists \bar{z} \in P)(\forall \bar{w} \in P)\left(\varphi(\bar{y}, \bar{w}) \leftrightarrow \psi_{\varphi}(\bar{z}, \bar{w})\right) .
$$

Пусть $Q_{1}, \ldots, Q_{n}$ - произвольные кванторные символы. Тогда

$$
\begin{aligned}
\left(\mathrm{Q}_{1} w_{1}\right. & \in P) \ldots\left(\mathrm{Q}_{n} w_{n} \in P\right) \varphi(\bar{y}, \bar{w}) \\
& \Leftrightarrow(\exists \bar{z} \in P)\left((\forall \bar{w} \in P)\left(\varphi(\bar{y}, \bar{w}) \leftrightarrow \psi_{\varphi}(\bar{z}, \bar{w})\right)\right. \\
& \left.\wedge\left(\mathrm{Q}_{1} w_{1} \in P\right) \ldots\left(\mathrm{Q}_{n} w_{n} \in P\right) \psi_{\varphi}(\bar{z}, \bar{w})\right) .
\end{aligned}
$$

Поскольку $\psi_{\varphi}-$ порядковая формула, $\bar{z} \in I$, а порядок на $I$ плотный, то все кванторы в формуле

$$
\left(\mathrm{Q}_{1} w_{1} \in P\right) \ldots\left(\mathrm{Q}_{n} w_{n} \in P\right) \psi_{\varphi}(\bar{z}, \bar{w})
$$

элиминируются.

СледСТВИЕ 5. Если система $\mathfrak{A}$ является әффективно I-сводимой, то приведенное в лемме 4 построение әффективно.

ДокАЗАТЕльство. Формула $\psi_{\varphi}$ строится эффективно, а теория плотного линейного порядка без концевых элементов допускает эффективную элиминацию кванторов.

Итак, мы имеем $P$-ограниченную формулу вида

$$
(\exists \bar{z} \in P)(\forall \bar{w} \in P) \varphi(x, \bar{y}, \bar{z}, \bar{w})
$$

где $\varphi$ - формула в сигнатуре $\Sigma$. Очевидно, что формула

$$
(\exists x)(\exists \bar{z} \in P)(\forall \bar{w} \in P) \varphi(x, \bar{y}, \bar{z}, \bar{w})
$$

эквивалентна формуле

$$
(\exists \bar{z} \in P)(\exists x)(\forall \bar{w} \in P) \varphi(x, \bar{y}, \bar{z}, \bar{w}) .
$$

Следовательно, достаточно показать, что любая формула вида

$$
(\exists x)(\forall \bar{w} \in P) \varphi(x, \bar{y}, \bar{w})
$$

эквивалентна ограниченной. 


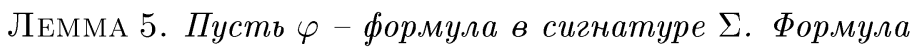

$$
(\exists x)(\forall \bar{w} \in P) \varphi\left(x, \bar{y}_{0}, \bar{w}\right)
$$

истинна в системе $(\mathfrak{A}, I)$ тогда и только тогда, когда для любого конечного множества $S \subseteq I$ существует такое $x_{S} \in A$, что для любого набора $\bar{s} \in S$

$$
(\mathfrak{A}, I) \models \varphi\left(x_{S}, \bar{y}_{0}, \bar{s}\right) .
$$

ДоказАтельство. Необходимость утверждения очевидна. Докажем достаточность.

Рассмотрим множество формул

$$
Y=\left\{\varphi\left(x, \bar{y}_{0}, \bar{d}\right): \bar{d} \in I\right\} .
$$

Множество $Y$ конечно совместно, потому что если $Y_{1} \subseteq Y$ - конечное подмножество $Y$, то в качестве $S$ можно взять все элементы всех наборов $\bar{d}$ из формул множества $Y_{1}$, и тогда, по условию, найдется $x_{S}$, удовлетворяющий всем формулам из $Y_{1}$. Следовательно, $Y$ - конечно совместное множество формул над множеством констант

$$
C=I \cup\left\{y_{0}^{(1)}, \ldots, y_{0}^{(|\bar{y}|)}\right\} .
$$

Очевидно, что множество $C$ имеет мошность, меньшую, чем $|I|^{+}$. Поскольку система $\mathfrak{A}$ является $|I|^{+}$-насьшенной, то множество $Y$ реализуется на некотором элементе $x_{0}$. Следовательно, будем иметь

$$
(\mathfrak{A}, I) \models \varphi\left(x_{0}, \bar{y}_{0}, \bar{d}\right)
$$

для всех $\bar{d} \in I$, из чего следует

$$
(\mathfrak{A}, I) \models(\exists x)(\forall \bar{w} \in P) \varphi\left(x, \bar{y}_{0}, \bar{w}\right),
$$

что и требовалось доказать.

\section{$\S 5 . \quad(k, l)$-формулы}

Итак, мы рассматриваем произвольную формулу $\varphi(x, \bar{y}, \bar{w})$ в сигнатуре $\Sigma$. Для нее существует порядковая формула $\psi_{\varphi}(\bar{z}, \bar{w})$ такая, что для всяких $x_{0}, \bar{y}_{0} \in A$ сушествует набор $\bar{c}_{\varphi}\left(x_{0}, \bar{y}_{0}\right) \in I$ такой, что

$$
(\mathfrak{A}, I) \models(\forall \bar{w} \in P)\left(\varphi\left(x_{0}, \bar{y}_{0}, \bar{w}\right) \leftrightarrow \psi_{\varphi}\left(\bar{c}_{\varphi}\left(x_{0}, \bar{y}_{0}\right), \bar{w}\right)\right) .
$$

Пусть $L=\left|\bar{c}_{\varphi}(x, \bar{y})\right|, N=|\bar{w}|$.

ОПРЕДЕЛЕНИЕ 7 . Пусть $k \geqslant 0, l \geqslant 1-$ натуральные числа. Через $\langle v, \bar{w}\rangle_{i}$ мы обозначаем набор, который получается из набора $\bar{w}$, если в него подставить элемент $v$ вместо $i$-го элемента $\bar{w}$. Обобщенной $(k, l)$-формулой мы будем называть формулу $\tilde{\varphi}(x, \bar{y}, \bar{u}, \bar{v})$, где $|\bar{v}|=k$, такую, что:

1) элементы наборов $\bar{u}$ и $\bar{v}$ образуют строго возрастающие последовательности, которые не содержат обших элементов; 
2) между двумя соседними элементами $\bar{v}$ находится или один элемент набора $\bar{u}$, или не менее $l$ элементов $\bar{u}$; если некоторые элементы $\bar{u}$ находятся левее $v_{1}$ (правее $v_{k}$ или если $k=0$ ), то на них распространяется то же требование: либо должен сушествовать один такой элемент, либо их должно быть не меньше $l$;

$3)$ для любого набора $\bar{w}$ длины $N$, составленного из элементов набора $\bar{u}$, выполнена формула $\varphi(x, \bar{y}, \bar{w})$;

4 ) для каждого элемента $v$ набора $\bar{v}$ существуют набор $\bar{w}$ длины $N$, составленный из элементов $\bar{u}$, и номер $i \in[1, N]$, для которых выполнено $\neg \varphi\left(x, \bar{y},\langle v, \bar{w}\rangle_{i}\right)$.

Части набора $\bar{u}$, которые располагаются между двумя соседними элементами набора $\bar{v}$ (а также те, которые меньше $v_{1}$ или больше $v_{k}$ ), будем для краткости называть сериями. Таким образом, в обобщенной $(k, l)$-формуле каждая серия состоит или из одного элемента (такие серии мы будем называть точечнымми), или из не менее $l$ элементов. Если все неточечные серии имеют длину ровно $l$, то такую формулу будем называть просто $(k, l)$-формулой.

Через $\bar{u}_{i}$ обозначим серию набора $\bar{u}$, расположенную между $v_{i}$ и $v_{i+1}$, а через $u_{i}^{(j)}-j$-й элемент этой серии.

Через $\operatorname{Set}(\bar{r})$ здесь и далее обозначим множество, составленное из всех элементов набора $\bar{r}$.

Следующие лемма и следствие из нее являются ключевыми в доказательстве. Они устанавливают, что если обобщенная $(k, l)$-формула выполнима, то $k$ не может быть сколь угодно большим.

ЛЕмма 6. Если $k \geqslant N+2$, обобщенная $(k, l)$-формула $\tilde{\varphi}(x, \bar{y}, \bar{u}, \bar{v})$ истинна в $(\mathfrak{A}, I)$ на әлементах $\left(x_{0}, \bar{y}_{0}, \bar{a}, \bar{b}\right), \bar{a} \in I, \bar{b} \in I, u i \leqslant k-N-1$, то в каждом промехутке вида $\left[b_{i}, a_{i+N-1}^{(1)}\right]$ есть хотя би один әлемент набора $\bar{c}_{\varphi}\left(x_{0}, \bar{y}_{0}\right)$.

ДокАЗАТЕльство. Пусть обобщенная $(k, l)$-формула $\tilde{\varphi}(x, \bar{y}, \bar{u}, \bar{v})$ истинна в $(\mathfrak{A}, I)$ на элементах $\left(x_{0}, \bar{y}_{0}, \bar{a}, \bar{b}\right), \bar{a} \in I, \bar{b} \in I$. Пусть $b_{i}$ - произвольный элемент набора $\bar{b}, i \leqslant k-N-1$. Выберем набор $\bar{d} \in \operatorname{Set}(\bar{a})$ такой, что

$$
(\mathfrak{A}, I) \models \neg \varphi\left(x_{0}, \bar{y}_{0},\left\langle b_{i}, \bar{d}\right\rangle_{j}\right)
$$

для некоторого $j$.

Построим новый набор $\bar{d}^{*}$ из набора $\left\langle b_{i}, \bar{d}\right\rangle_{j}$ следуюшим образом. Элемент $b_{i}$ заменим на $a_{i}^{(1)}$. Если в наборе $\left\langle b_{i}, \bar{d}\right\rangle_{j}$ элемент $a_{i}^{(1)}$ уже встречался, то заменим его на следующий за ним по порядку элемент из $\operatorname{Set}(\bar{a})$ и т. д. Мы произведем не больше $N$ замен, следовательно, все новые элементы $\bar{d}^{*}$ не превосходят $a_{i+N-1}^{(1)}$. Очевидно, что наборы $\bar{d}^{*}$ и $\left\langle b_{i}, \bar{d}\right\rangle_{j}$ одинаково упорядочены, и вне отрезка $\left[b_{i}, a_{i+N-1}^{(1)}\right]$ они совпадают.

Поскольку $\bar{d}^{*} \in \operatorname{Set}(\bar{a})$, то

$$
(\mathfrak{A}, I) \models \varphi\left(x, \bar{y}_{0}, \bar{d}^{*}\right) .
$$

Согласно следствию 4 это и означает наличие хотя бы одного элемента набора $\bar{c}_{\varphi}\left(x_{0}, \bar{y}_{0}\right)$ на указанном отрезке.

СЛЕДСТВИЕ 6. Существует константа $R$ такая, что для всех $k>R$ все обобщенные $(k, l)$-формуль $\tilde{\varphi}(x, \bar{y}, \bar{u}, \bar{v})$ не выполнимы ни на каких наборах $\left(x_{0}, \bar{y}_{0}, \bar{a}, \bar{b}\right)$, если $\bar{a} \in I, \bar{b} \in I$. 
ДоКАЗАТЕЛЬСТВО. Возьмем

$$
R=N(L+1) .
$$

Предположим, что $k \geqslant R+1$ и некоторая $(k, l)$-формула $\tilde{\varphi}$ выполнима на некотором наборе указанного вида. Согласно лемме на всяком отрезке вида $\left[b_{i}, a_{i+N-1}^{(1)}\right]$ имеется элемент набора $\bar{c}_{\varphi}\left(x_{0}, \bar{y}_{0}\right)$. Таким образом, на каждом из $L+1$ отрезков

$$
\left[b_{1}, a_{N}^{(1)}\right],\left[b_{N+1}, a_{2 N}^{(1)}\right], \ldots,\left[b_{L N+1}, a_{(L+1) N}^{(1)}\right]
$$

сушествует не меньше одного элемента набора $\bar{c}_{\varphi}\left(x_{0}, \bar{y}_{0}\right)$. Но эти отрезки не пересекаются, а различных элементов в наборе $\bar{c}_{\varphi}\left(x_{0}, \bar{y}_{0}\right)$ не больше $L$. Противоречие.

ОПРЕдЕЛЕНИЕ 8 . Пусть $\bar{a}_{i}-$ некоторая серия. Тогда через $\left[\bar{a}_{i}\right]$ мы обозначаем отрезок, в точности покрываюший все элементы этой серии, т. е. если

$$
\bar{a}_{i}=\left(a_{i}^{(1)}, \ldots, a_{i}^{(l)}\right),
$$

TO

$$
\left[\bar{a}_{i}\right]=\left[a_{i}^{(1)}, a_{i}^{(l)}\right] .
$$

Если $\tilde{\varphi}(x, \bar{y}, \bar{u}, \bar{v})$ - обобщенная формула, а $\bar{a}-$ некоторый набор, имеюший такую же длину, как $\bar{u}$, и упорядоченньй соответствующим образом, то через $[\bar{a}]_{\tilde{\varphi}}$ мы будем обозначать объединение всех отрезков $\left[\bar{a}_{i}\right]$, где $\bar{a}_{i}-$ все серии, на которые разбивается набор $\bar{a}$ в формуле $\tilde{\varphi}$.

На множестве (обобшенных) $(k, l)$-формул введем отношение "быть расширением".

ОПРЕДЕЛЕНИЕ 9. Формулу (обобщенную формулу) $\tilde{\varphi}_{i}\left(x, \bar{y}, \bar{u}^{\prime}, \bar{v}^{\prime}\right)$ мы называем расширением формуль (обобщенной формуль) $\tilde{\varphi}_{j}\left(x, \bar{y}, \bar{u}^{\prime \prime}, \bar{v}^{\prime \prime}\right)$, если для наборов $\left(\bar{a}^{\prime}, \bar{b}^{\prime}\right) \in I$ и $\left(\bar{a}^{\prime \prime}, \bar{b}^{\prime \prime}\right) \in I$, которые упорядочены по требованиям формул $\tilde{\varphi}_{i}$ и $\tilde{\varphi}_{j}$ соответственно, существует сохраняющее порядок разнозначное отображение

$$
f: \operatorname{Set}\left(\bar{a}^{\prime \prime}\right) \cup \operatorname{Set}\left(\bar{b}^{\prime \prime}\right) \rightarrow\left[\bar{a}^{\prime}\right]_{\tilde{\varphi}_{i}} \cup \operatorname{Set}\left(\bar{b}^{\prime}\right)
$$

такое, что

$$
f\left[\operatorname{Set}\left(\bar{b}^{\prime \prime}\right)\right] \subseteq \operatorname{Set}\left(\bar{b}^{\prime}\right),
$$

и для каждой серии $\bar{a}_{i}^{\prime \prime}$ сушествует серия $\bar{a}_{j}^{\prime}$, для которой

$$
f\left[\operatorname{Set}\left(\bar{a}_{i}^{\prime \prime}\right)\right] \subseteq\left[\bar{a}_{j}^{\prime}\right] .
$$

В частности, каждая (обобщенная) формула является расширением самой себя. Очевидно также, что отношение "быть расширением" транзитивно, так как $I$ плотно упорядочено.

Зафиксируем константу $R$, сушествование которой следует из следствия 6 . Пусть

$$
Q=\max \{N(2 L+1), R(N-1)+2\} .
$$

ОПРЕДЕЛЕНИЕ $10 . k$ - формулой (обобщенной) мы будем называть $(k, Q)$-формулу (обобщенную), в которой существует хотя бы одна неточечная серия (заметим, что $Q \geqslant 2$, поэтому определение корректно). 
Очевидно, что для каждого $k \geqslant 1$ сушествует $9 \cdot 2^{k-1}-4$ различных $k$-формул (каждая из $k-1$ "внутренних" серий $\bar{u}$ или является точечной, или не является таковой, серии левее $v_{1}$ или правее $v_{k}$ могут отсутствовать, быть точечными или иметь длину $Q$, четыре формулы содержат только точечные серии). Для $k=0$ сушествует только одна $k$-формула. Всего различных выполнимых $k$-формул не больше, чем

$$
K=1+\sum_{k=1}^{R}\left(9 \cdot 2^{k-1}-4\right)
$$

т. е. их количество заранее ограничено константой $K$.

Отношение "быть расширением" является частичным порядком на множестве $k$-формул. Будем считать, что для $k \in[0, R]$ все $k$-формулы $\tilde{\varphi}_{i}$ упорядочены по $i$ так, что если $\tilde{\varphi}_{i}$ является расширением $\tilde{\varphi}_{j}$, то $i \geqslant j$. Пусть $\tilde{\varphi}_{0}, \ldots, \tilde{\varphi}_{K-1}-$ все эти формулы. Поскольку мы не рассматриваем формул, которые содержат только точечные серии, то $\tilde{\varphi}_{0}$ не содержит никаких $v$ и содержит одну серию $\bar{u}$ длины $Q$.

Из следующих лемм вытекает, что при некоторых преобразованиях выполнимые (обобщенные) $k$-форомлы остаются выполнимыми, но приобретают необходимые нам свойства.

ЛЕмма 7. Пусть $\tilde{\varphi}_{i}(x, \bar{y}, \bar{u}, \bar{v})$ - обобщенная $k$-формула, которая выполняется на $\left(x_{0}, \bar{y}_{0}, \bar{a}, \bar{b}\right), \bar{a} \in I, \bar{b} \in I$. Тогда в любую неточечную серию $\bar{a}_{i}$ можно добавить некоторый элемент $a^{*} \in I$ и формула, полученная аналогичным добавлением новой переменной $u^{*}$, будет истинной на вновь полученном наборе.

ДокаЗАТЕЛьство. Рассмотрим произвольную неточечную серию $\bar{a}_{i}$ из $\bar{a}$. Она содержит не менее $N(2 L+1)$ элементов. Поскольку число различных элементов в наборе $\bar{c}_{\varphi}\left(x_{0}, \bar{y}_{0}\right)$ не больше $L$, то существует не более $2 L+1$ различных способов расположения каждой точки относительно этого набора. Следовательно, среди $Q$ элементов серии $\bar{a}_{i}$ есть как минимум $N$ различных элементов $a_{1}^{\prime}<\cdots<a_{N}^{\prime}$, которые одинаково расположены относительно $\bar{c}_{\varphi}\left(x_{0}, \bar{y}_{0}\right)$. Рассмотрим любой новый элемент $a^{*} \in\left[a_{1}^{\prime}, a_{N}^{\prime}\right] \cap I$, вставим его в $\bar{a}$ и получим $\bar{a}^{*}$. Построим новую формулу $\tilde{\varphi}_{j}\left(x, \bar{y}, \bar{u}^{*}, \bar{v}\right)$ аналогичньм образом, добавив новую переменную $u^{*}$ к уже существующим $\bar{u}$. Пусть набор $\bar{d}^{*}$ составлен из элементов нового множества $\operatorname{Set}\left(\bar{a}^{*}\right)$. Тогда он имеет такой же порядковый тип над $\bar{c}_{\varphi}\left(x_{0}, \bar{y}_{0}\right)$, как и некоторый набор $\bar{d}$, которьй составлен из элементов старого множества $\operatorname{Set}(\bar{a})$. Следовательно,

$$
\psi_{\varphi}\left(\bar{c}_{\varphi}\left(x_{0}, \bar{y}_{0}\right), \bar{d}^{*}\right) \Leftrightarrow \psi_{\varphi}\left(\bar{c}_{\varphi}\left(x_{0}, \bar{y}_{0}\right), \bar{d}\right)
$$

Из этого получаем истинность $\varphi\left(x_{0}, \bar{y}_{0}, \bar{d}^{*}\right)$. Следовательно, формула $\tilde{\varphi}_{j}$ истинна на $\left(x_{0}, \bar{y}_{0}, \bar{a}^{*}, \bar{b}\right)$, если формула $\tilde{\varphi}_{i}$ была истинна на наборе $\left(x_{0}, \bar{y}_{0}, \bar{a}, \bar{b}\right)$.

Итерируя применение этой леммы, получаем

СЛЕДСТВИЕ 7. Пусть $\tilde{\varphi}_{i}$ - обобщенная $k$-формула и выполнена формула $\tilde{\varphi}_{i}\left(x_{0}, \bar{y}_{0}, \bar{a}, \bar{b}\right), \bar{a} \in I, \bar{b} \in I$. Тогда все неточечные серии можно увеличить до произвольной длины таким образом, что обобщенная $k$-формула, полученная аналогичным увеличением количества переменных $\bar{u}$, будет истинной на вновь полученном наборе. 
ЛЕмма 8. Пусть обобщенная $k$-формула $\tilde{\varphi}_{i}(x, \bar{y}, \bar{u}, \bar{v})$ выполнена на наборе $\left(x_{0}, \bar{y}_{0}, \bar{a}, \bar{b}\right), \bar{a} \in I, \bar{b} \in I$. Пусть $G \subseteq I$ - конечное множество и для любого $\bar{g} \in G \cup \operatorname{Set}(\bar{a})$ выполнено $\varphi\left(x_{0}, \bar{y}_{0}, \bar{g}\right)$. Тогда существуют обобщенная $k^{\prime}$-формула $\tilde{\varphi}_{j}-$ расширение $\tilde{\varphi}_{i}-$ и наборы $\bar{a}^{\prime} \in I, \bar{b}^{\prime} \in I$ такие, что:

1) выполнена формула $\tilde{\varphi}_{j}\left(x_{0}, \bar{y}_{0}, \bar{a}^{\prime}, \bar{b}^{\prime}\right)$;

2) $\operatorname{Set}(\bar{a}) \cup G \subseteq \operatorname{Set}\left(\bar{a}^{\prime}\right)$.

ДоКАЗАТЕЛЬСТво. Сначала включим каждый элемент $g$ множества $G$ в подходящую серию $\bar{a}_{i}$, т. е. такую, чтобы между элементами $\bar{a}_{i}$ и $g$ не было никаких $b$. Возможно, что придется создать новые серии, если какой-то элемент $g \in G$ меньше $b_{1}$ или больше $b_{k}$, а соответствуюшие серии отсутствуют.

После этих действий может оказаться, что появились новые неточечные серии длины, меньшей $Q$, и мы должны избавиться от таких серий. Будем делать это пошагово.

На каждом шаге рассмотрим произвольную "короткую" серию $\bar{a}_{i}$. Если в $\left[\bar{a}_{i}\right]$ сушествует элемент $h \in I$ такой, что для некоторого набора $\bar{d} \in \operatorname{Set}(\bar{a})$ имеет место $\neg \varphi\left(x_{0}, \bar{y}_{0},\langle h, \bar{d}\rangle_{n}\right)$ для некоторого $n$, то берем такое $h$ в качестве нового элемента $b$, а серию $\bar{a}_{i}$ расщепляем на две более короткие. Если же таких элементов нет, то любой элемент из $\left[\bar{a}_{i}\right] \cap I$ можно добавить к серии $\bar{a}_{i}$. Аналогичные изменения производим и с формулой: в первом случае добавляем новую переменную $v$, во втором - новое $u$.

Поскольку количество элементов $v$ в любой выполнимой обобщенной $k$-формуле не может быть больше $R$, то количество шагов, на которых в формулу добавляется новое $v$, ограничено этим числом. Следовательно, рано или поздно любую "короткую" серию мы сможем довести до необходимой длины $Q$ или эта серия будет разбита на точечные.

Очевидно, что новая формула будет расширением старой, так как все серии старой формулы остались, неточечные серии старой формулы только увеличили свою длину и, возможно, появились новые серии.

ЛЕмма 9. Пусть обобщенная $k$-формула $\tilde{\varphi}_{i}(x, \bar{y}, \bar{u}, \bar{v})$ выполнена на наборе $\left(x_{0}, \bar{y}_{0}, \bar{a}, \bar{b}\right), \bar{a} \in I, \bar{b} \in I$. Тогда существуют $k^{\prime}$-формула $\tilde{\varphi}_{j}-$ расширение $\tilde{\varphi}_{i}-$ и наборы $\bar{a}^{\prime} \in I, \bar{b}^{\prime} \in I$ такие, что:

1) выполнено $\tilde{\varphi}_{j}\left(x_{0}, \bar{y}_{0}, \bar{a}^{\prime}, \bar{b}^{\prime}\right)$;

2) $\operatorname{Set}(\bar{a}) \subseteq\left[\bar{a}^{\prime}\right]_{\tilde{\varphi}_{j}}$;

3) для любого набора $\bar{e} \in\left[\bar{a}^{\prime}\right] \tilde{\varphi}_{j} \cap I$ выполнено $\varphi\left(x_{0}, \bar{y}_{0}, \bar{e}\right)$.

ДокАЗАТЕЛЬСТво. Доказательство будем проводить в два этапа: на первом этапе мы построим обобщенную $k^{\prime}$-формулу, для которой будут выполнены условия 1)-3), а на втором - "сократим" эту обобщенную $k^{\prime}$-формулу до $k^{\prime}$-формулы.

Если указанные условия выполняются для $\tilde{\varphi}_{j}=\tilde{\varphi}_{i}, k^{\prime}=k, \bar{a}^{\prime}=\bar{a}, \bar{b}^{\prime}=\bar{b}$, то переходим ко второй части доказательства.

Допустим, что это не так, и условие 3 ) не выполняется. Заметим, что условия 1) и 2) выполнены. Покажем, как построить $\tilde{\varphi}_{j}, \bar{a}^{\prime}$ и $\bar{b}^{\prime}$, чтобы условие 3 ) стало выполняться.

Пусть для некоторого $\bar{e} \in[\bar{a}]_{\tilde{\varphi}_{i}} \cap I$ выполнено $\neg \varphi\left(x_{0}, \bar{y}_{0}, \bar{e}\right)$. Поочередно будем добавлять в $\bar{a}$ те элементы $e$ из $\bar{e}$, для которых формула $\varphi\left(x_{0}, \bar{y}_{0},\langle e, \bar{d}\rangle_{n}\right)$ истинна для любых $\bar{d} \in \operatorname{Set}(\bar{a})$ и всех $n$, в формулу вносим аналогичные изменения. Очевидно, что все элементы из $\bar{e}$ мы добавить не сможем, следовательно, на некотором 
шаге для любого $e$ из оставшихся элементов $\bar{e}$ существует такое $\bar{d} \in \operatorname{Set}(\bar{a})$, что вьполняется $\neg \varphi\left(x_{0}, \bar{y}_{0},\langle e, \bar{d}\rangle_{n}\right)$ для некоторого $n$.

Согласно лемме 7 и следствию 7 можно считать, что между двумя элементами $\bar{b}$ находится или один элемент $\bar{a}$, или не меньше, чем $Q+1$ элементов.

Если существует $h \in I$, отделеннцй от существующих элементов $\bar{b}$ ровно одним элементом $\bar{a}$, и для $h$ сушествует $\bar{d} \in \operatorname{Set}(\bar{a})$ такой, что $\neg \varphi\left(x_{0}, \bar{y},\langle h, \bar{d}\rangle_{n}\right)$ для некоторого $n$, то этот элемент добавим в качестве нового $b$. Получим новые наборы $\bar{a}^{\prime \prime}$ и $\bar{b}^{\prime \prime}$, обобшенную формулу $\tilde{\varphi}_{m}$, которая выполняется на этих наборах, и перейдем к поиску новых возможных $b$. Заметим только, что все элементы старого $\bar{a}$ попадают в новое $\bar{a}^{\prime \prime}$ и все неточечные серии сохранили достаточную длину. Следовательно, формула $\tilde{\varphi}_{m}-\operatorname{pacширение~} \tilde{\varphi}_{i}, \operatorname{Set}(\bar{a}) \subseteq \operatorname{Set}\left(\bar{a}^{\prime \prime}\right)$.

Предположим теперь, что любой $h \in I$, отделенньй от $\bar{b}$ ровно одним элементом $\bar{a}$, удовлетворяет условию $\varphi\left(x_{0}, \bar{y}_{0},\langle h, \bar{d}\rangle_{n}\right)$ для любых $\bar{d} \in \operatorname{Set}(\bar{a})$ и всех $n$. В частности, это означает, что все оставшиеся элементы $e$ отделены от каждого из элементов $b$ не менее чем двумя элементами $\bar{a}$. Предположим, что существует такой $h^{\prime} \in I \cap[\bar{a}]_{\tilde{\varphi}_{i}}$, для которого найдется $\bar{d} \in \operatorname{Set}(\bar{a})$ такой, что $\neg \varphi\left(x_{0}, \bar{y},\left\langle h^{\prime}, \bar{d}\right\rangle_{n}\right)$ для некоторого $n$, и $h^{\prime}$ отделен от всех элементов $\bar{b}$ не менее чем $Q$ элементами $\bar{a}$. В этом случае добавляем этот $h^{\prime}$ в формулу в качестве нового $b$, а серию, внутри которой он находится, расщепляем на две неточечные, то же самое делаем с формулой. Очевидно, что новые серии будут иметь достаточную длину, новая формула станет расширением старой и $\operatorname{Set}(\bar{a}) \subseteq \operatorname{Set}\left(\bar{a}^{\prime \prime}\right)$.

Если ни одно из двух предыдущих условий не выполнено, то для каждого оставшегося элемента $e$ существует соседний с ним элемент $b$, отделенный от $e$ менее чем $Q$, но не менее чем двумя элементами $\bar{a}$. Вставим в этот промежуток новый элемент $h$ набора $\bar{a}$, для которого выполняется $\varphi\left(x_{0}, \bar{y}_{0},\langle h, \bar{d}\rangle_{n}\right)$ при любых $\bar{d} \in$ $\operatorname{Set}(\bar{a})$ и всех $n$. Такой $h$, как мы уже доказали, сушествует. Для нового набора $\bar{a}^{\prime \prime}$ будем иметь $\operatorname{Set}(\bar{a}) \subseteq \operatorname{Set}\left(\bar{a}^{\prime \prime}\right)$. Заметим, что мы не будем выполнять более $2 Q N$ шагов такого вида подряд, так как когда мы их выполним, будут созданы условия для выполнения шага предыдушего вида - каждый элемент $e$ окажется отделен от соседних $b$ не менее чем $Q$ элементами $\bar{a}$.

Поскольку на каждом шаге мы получаем выполнимую формулу, то $\left|\bar{b}^{\prime \prime}\right| \leqslant R$. Отсюда следует, что общее количество шагов ограничено константой $(2 Q N+1) R$. Следовательно, на каком-либо этапе мы не сможем найти очередного $\bar{e} \in$ $\left[\bar{a}^{\prime \prime}\right]_{\tilde{\varphi}_{m}} \cap I$, что и будет означать выполнение условия 3$)$.

Мы получили обобщенную $k^{\prime}$-формулу $\tilde{\varphi}_{m}$, которая удовлетворяет условиям 1)-3). Теперь необходимо преобразовать ее в $k^{\prime}$-формулу, удалив "лишние" элементы из $\bar{a}^{\prime \prime}$. Элементы $\bar{b}^{\prime \prime}$ оставим без изменений: $\bar{b}^{\prime}=\bar{b}^{\prime \prime}$.

Для каждого элемента $b$ из набора $\bar{b}^{\prime}$ требуется не более $N-1$ элемента набора $\bar{a}^{\prime \prime}-$ те $\bar{d} \in \operatorname{Set}\left(\bar{a}^{\prime \prime}\right)$, вместе с которыми получаем $\neg \varphi\left(x_{0}, \bar{y}_{0},\langle b, \bar{d}\rangle_{n}\right)$ для некоторого $n\left(d_{n}\right.$, естественно, включать не нужно). Поскольку общее количество элементов $\bar{b}$ не превышает $R$, то для истинности некоторой $k^{\prime}$-формулы необходимо не больше

$$
R(N-1) \leqslant Q-2
$$

элементов $\bar{a}^{\prime \prime}$. Для каждой серии оставляем ровно столько элементов, оставляем также по два крайних элемента каждой серии, а остальные элементы из середины серий $\bar{a}^{\prime \prime}$ можно удалить. Получим $k^{\prime}$-формулу $\tilde{\varphi}_{j}$ и набор $\bar{a}^{\prime}$, для которых 
вьполнено $\left[\bar{a}^{\prime}\right]_{\tilde{\varphi}_{j}}=\left[\bar{a}^{\prime \prime}\right]_{\tilde{\varphi}_{m}}$. Следовательно, $\operatorname{Set}\left(\bar{a}^{\prime \prime}\right) \subseteq\left[\bar{a}^{\prime}\right]_{\tilde{\varphi}_{j}}$, а так как $\operatorname{Set}(\bar{a}) \subseteq$ $\operatorname{Set}\left(\bar{a}^{\prime \prime}\right)$, то $\operatorname{Set}(\bar{a}) \subseteq\left[\bar{a}^{\prime}\right]_{\tilde{\varphi}_{j}}$.

Итак, мы получили новые $\bar{a}^{\prime}, \bar{b}^{\prime}$ и $\tilde{\varphi}_{j}$ такие, что $\tilde{\varphi}_{j}\left(x_{0}, \bar{y}_{0}, \bar{a}^{\prime}, \bar{b}^{\prime}\right)$.

Очевидно, что $k^{\prime}$-формула $\tilde{\varphi}_{j}$ является расширением обобщенной $k$-формулы $\tilde{\varphi}_{i}$.

Комбинируя две предыдущие леммы, получаем

ЛЕмма 10. Пусть $k$-формула $\tilde{\varphi}_{i}(x, \bar{y}, \bar{u}, \bar{v})$ выполнена на наборе $\left(x_{0}, \bar{y}_{0}, \bar{a}, \bar{b}\right)$, $\bar{a} \in I, \bar{b} \in I$. Пусть $G \subseteq I$ - конечное множество и для любого $\bar{g} \in G \cup \operatorname{Set}(\bar{a})$ выполнено $\varphi\left(x_{0}, \bar{y}, \bar{g}\right)$. Тогда существуют $k^{\prime}$-формула $\tilde{\varphi}_{j}-$ расширение $\tilde{\varphi}_{i}-u$ наборы $\bar{a}^{\prime} \in I, \bar{b}^{\prime} \in I$ такие, что:

1) выполнено $\tilde{\varphi}_{j}\left(x_{0}, \bar{y}_{0}, \bar{a}^{\prime}, \bar{b}^{\prime}\right)$;

2) $\operatorname{Set}(\bar{a}) \cup G \subseteq\left[\bar{a}^{\prime}\right]_{\tilde{\varphi}_{j}}$;

3) для любого набора $\bar{e} \in\left[\bar{a}^{\prime}\right] \tilde{\varphi}_{j} \cap I$ выполнено $\varphi\left(x_{0}, \bar{y}_{0}, \bar{e}\right)$.

ДоКАЗАТЕЛЬСтво. Сначала воспользуемся леммой 8 , получим обобщенную $k^{\prime \prime}$-формулу $\tilde{\varphi}_{m}\left(x_{0}, \bar{y}_{0}, \bar{a}^{\prime \prime}, \bar{b}^{\prime \prime}\right)$ такую, что $G \cup \operatorname{Set}(\bar{a}) \subseteq \operatorname{Set}\left(\bar{a}^{\prime \prime}\right)$.

После этого по лемме 9 получим искомую $k^{\prime}$-формулу $\tilde{\varphi}_{j}$ и наборы $\bar{a}^{\prime}, \bar{b}^{\prime}$.

СлЕДСТвИЕ 8. Если в условиях леммы 10 для некоторого $\bar{e} \in[\bar{a}]_{\tilde{\varphi}_{i}} \cap I$ выполнено $\neg \varphi\left(x_{0}, \bar{y}_{0}, \bar{e}\right)$, то для построенной формулы $\tilde{\varphi}_{j}$ имеет место $j>i$.

\section{§6. Основные результаты}

Теперь можно построить $P$-ограниченную формулу, которая будет эквивалентна формуле

$$
(\exists x)(\forall \bar{w} \in P) \varphi(x, \bar{y}, \bar{w}) .
$$

Определим формулы $\tilde{\varphi}_{i}^{\prime}(x, \bar{y}, \bar{a}, \bar{b})$ обратной индукцией по $i$. Если $i=K-1$, то $\tilde{\varphi}_{i}^{\prime} \sim \tilde{\varphi}_{i}$. Пусть для $j>i$ формулы $\tilde{\varphi}_{j}^{\prime}$ определены. По индукции считаем, что формулы $(\exists x) \tilde{\varphi}_{j}^{\prime}$ для $j>i$ эквивалентны $P$-ограниченным, следовательно, для каждой формулы $\tilde{\varphi}_{j}^{\prime}$ сушествует порядковая бескванторная формула $\psi_{j}$ такая, что

$$
(\forall \bar{y})\left(\exists \bar{f}_{j} \in P\right)(\forall \bar{a}, \bar{b} \in P)\left(\left((\exists x) \tilde{\varphi}_{j}^{\prime}(x, \bar{y}, \bar{a}, \bar{b})\right) \leftrightarrow \psi_{j}\left(\bar{f}_{j}, \bar{a}, \bar{b}\right)\right) .
$$

Если длина набора $\bar{f}_{j}$ равна $n$, то длину набора $\bar{e}_{j}$ полагаем равной $(n+1)(R+2)$, а формула $\Theta_{j}\left(\bar{f}_{j}, \bar{e}_{j}\right)$ утверждает, что в каждом из открытых промежутков, на которые разбивает $I$ набор $\bar{f}_{j}$ (будем называть их $\bar{f}_{j}$-промежутками), содержится как минимум $R+2$ различных элементов $\bar{e}_{j}$. Очевидно, можно считать, что $\Theta_{j}-$ порядковая бескванторная формула. Обозначим с помощью $\tilde{\varphi}_{i}^{\prime}$ следующую формулу:

$$
\begin{aligned}
& \tilde{\varphi}_{i}^{\prime}(x, \bar{y}, \bar{u}, \bar{v}) \sim\left(\exists \bar{f}_{K}, \ldots, \bar{f}_{i+1} \in P\right) \\
& \left(\left(\bigwedge_{j=i+1}^{K-1}\left(\forall \bar{u}_{j}, \bar{v}_{j} \in P\right)\left(\left((\exists x) \tilde{\varphi}_{j}^{\prime}\left(x, \bar{y}, \bar{u}_{j}, \bar{v}_{j}\right)\right) \leftrightarrow \psi_{j}\left(\bar{f}_{j}, \bar{u}_{j}, \bar{v}_{j}\right)\right)\right)\right. \\
& \quad \wedge\left(\exists \bar{e}_{K}, \ldots, \bar{e}_{i+1} \in P\right)\left(\left(\bigwedge_{j=i+1}^{K-1} \Theta_{j}\left(\bar{f}_{j}, \bar{e}_{j}\right)\right)\right. \\
& \left.\left.\quad \wedge\left(\bigwedge_{\bar{g} \in G_{i} \cup \operatorname{Set}(\bar{u})} \varphi(x, \bar{y}, \bar{g})\right) \wedge \tilde{\varphi}_{i}(x, \bar{y}, \bar{u}, \bar{v})\right)\right) .
\end{aligned}
$$


Здесь $G_{i}$ - множество, составленное из всех переменных наборов $\bar{f}_{K}, \bar{e}_{K}, \ldots, \bar{f}_{i+1}$, $\bar{e}_{i+1}$. Ясно, что формула $(\exists x) \tilde{\varphi}_{i}^{\prime}$ эквивалентна $P$-ограниченной.

Определим формулы $\Phi_{i}$ :

$$
\begin{aligned}
\Phi_{i}(\bar{y}) & \sim\left(\exists \bar{f}_{i} \in P\right)\left((\forall \bar{u}, \bar{v} \in P)\left(\left((\exists x) \tilde{\varphi}_{i}^{\prime}(x, \bar{y}, \bar{u}, \bar{v})\right) \leftrightarrow \psi_{i}\left(\overline{f_{i}}, \bar{u}, \bar{v}\right)\right)\right. \\
& \wedge\left(\exists \bar{e}_{i} \in P\right)\left(\Theta_{i}\left(\bar{f}_{i}, \bar{e}_{i}\right)\right. \\
& \left.\left.\wedge(\exists \bar{u}, \bar{v} \in P)(\exists x)(\underbrace{\left(\operatorname{Set}\left(\bar{f}_{i}, \bar{e}_{i}\right) \subseteq[\bar{u}]_{\tilde{\varphi}_{i}}\right) \wedge \tilde{\varphi}_{i}^{\prime}(x, \bar{y}, \bar{u}, \bar{v})}_{\Phi_{i}^{\prime}})\right)\right) .
\end{aligned}
$$

Сокращение $\operatorname{Set}\left(\bar{f}_{i}, \bar{e}_{i}\right) \subseteq[\bar{u}]_{\tilde{\varphi}_{i}}$, очевидно, можно записать в виде порядковой бескванторной формулы. Пусть

$$
\Phi \sim \bigvee_{j=0}^{K-1} \Phi_{j}
$$

Очевидно, что формулы $\Phi_{i}$ и, следовательно, $\Phi$ эквивалентны $P$-ограниченным.

СЛЕДСТВИЕ 9. Если система $\mathfrak{A}$ является әффективно I-сводимой, то формуль $\tilde{\varphi}_{i}^{\prime}, \Phi_{i}$ и $\Phi$ строятся по формуле $\varphi$ әффективно.

ДокАЗАТЕЛЬСТвО следует из того, что если $I$-сведение является эффективным, то константы $N, L, R, Q, K$ могут быть эффективно найдены по формуле $\varphi$.

ЛЕмма 11. Пусть выполнено $\Phi\left(\bar{y}_{0}\right)$, и пусть $i$ - максимальный номер, для которого выполнено $\Phi_{i}\left(\bar{y}_{0}\right)$. Пусть $\bar{f}_{i} \in I, \quad \bar{e}_{i} \in I, \quad x_{0}, \bar{a} \in I \quad u \bar{b} \in I-$ конкретные әлементыл, на которых выполняется формула $\Phi_{i}^{\prime}$. Тогда для любых $\bar{a}^{\prime} \in I$ и $\bar{b}^{\prime} \in I$, расположенных относительно $\bar{f}_{i}$ так жее, как $\bar{a} u \bar{b}$, существует такой $x_{0}^{\prime}$, что

$$
(\mathfrak{A}, I) \models \tilde{\varphi}_{i}^{\prime}\left(x_{0}^{\prime}, \bar{y}_{0}, \bar{a}^{\prime}, \bar{b}^{\prime}\right),
$$

и для любого $\bar{g} \in\left[\bar{a}^{\prime}\right]_{\tilde{\varphi}_{i}}$ выполняется $\varphi\left(x_{0}^{\prime}, \bar{y}_{0}, \bar{g}\right)$.

ДокАЗАТЕЛЬСтво. Поскольку

$$
(\mathfrak{A}, I) \models(\forall \bar{u}, \bar{v} \in P)\left(\left((\exists x) \tilde{\varphi}_{i}^{\prime}\left(x, \bar{y}_{0}, \bar{u}, \bar{v}\right)\right) \leftrightarrow \psi_{i}\left(\bar{f}_{i}, \bar{u}, \bar{v}\right)\right),
$$

то если взять любые $\bar{a}^{\prime}$ и $\bar{b}^{\prime}$, которые имеют такой же порядковьй тип над $\bar{f}_{i}$, что и исходные, то для этих $\bar{a}^{\prime}$ и $\bar{b}^{\prime}$ тоже найдется некоторый $x_{0}^{\prime}$, для которого выполнено $\tilde{\varphi}_{i}^{\prime}\left(x_{0}^{\prime}, \bar{y}_{0}, \bar{a}^{\prime}, \bar{b}^{\prime}\right)$. Из этого следует, что для любого набора $\bar{g} \in G_{i} \cup \operatorname{Set}\left(\bar{a}^{\prime}\right)$ имеет место $\varphi\left(x_{0}^{\prime}, \bar{y}_{0}, \bar{g}\right)$.

Рассмотрим любой $\bar{g} \in\left[\bar{a}^{\prime}\right]_{\tilde{\varphi}_{i}}$. Предположим, что для него выполнена формула $\neg \varphi\left(x_{0}^{\prime}, \bar{y}_{0}, \bar{g}\right)$. Тогда согласно лемме 10 можно построить $\tilde{\varphi}_{j}$ - расширение формулы $\tilde{\varphi}_{i}$ - такое, что:

1) выполнено $\tilde{\varphi}_{j}\left(x_{0}^{\prime}, \bar{y}_{0}, \bar{a}^{\prime \prime}, \bar{b}^{\prime \prime}\right)$;

2) $G_{i} \cup \operatorname{Set}\left(\bar{a}^{\prime}\right) \subseteq\left[\bar{a}^{\prime \prime}\right]_{\tilde{\varphi}_{j}}$;

3) для всех $\bar{g} \in\left[\bar{a}^{\prime \prime}\right]_{\tilde{\varphi}_{j}}$ выполнено $\varphi\left(x_{0}^{\prime}, \bar{y}_{0}, \bar{g}\right)$. 
Согласно следствию 8 имеем $j>i$. Рассмотрим произвольный элемент

$$
g \in G_{j} \cup \operatorname{Set}\left(\bar{f}_{j}, \bar{e}_{j}\right) \cup \operatorname{Set}\left(\bar{a}^{\prime \prime}\right) .
$$

Очевидно, что $G_{j} \cup \operatorname{Set}\left(\bar{f}_{j}, \bar{e}_{j}\right) \subseteq G_{i}$, так как $j>i$. Следовательно, $g$ является элементом множества $G_{i} \cup \operatorname{Set}\left(\bar{a}^{\prime \prime}\right)$. Но тогда $g \in\left[\bar{a}^{\prime \prime}\right]_{\tilde{\varphi}_{j}}$. Из этого получаем, что для любого $\bar{g} \in G_{j} \cup \operatorname{Set}\left(\bar{a}^{\prime \prime}\right)$ выполняется $\varphi\left(x_{0}^{\prime}, \bar{y}_{0}, \bar{g}\right)$, откуда следует истинность $\tilde{\varphi}_{j}^{\prime}\left(x_{0}^{\prime}, \bar{y}_{0}, \bar{a}^{\prime}, \bar{b}^{\prime}\right)$. Из этого получается истинность $\Phi_{j}\left(\bar{y}_{0}\right)$, что противоречит максимальности $i$.

Докажем, что $\Phi$ как раз и является $P$-ограниченной формулой, которая эквивалентна

$$
(\exists x)(\forall \bar{w} \in P) \varphi(x, \bar{y}, \bar{w}) .
$$

Лемма 12. Eсли

$$
(\mathfrak{A}, I) \models(\exists x)(\forall \bar{w} \in P) \varphi\left(x, \bar{y}_{0}, \bar{w}\right),
$$

$m o(\mathfrak{A}, I) \models \Phi\left(\bar{y}_{0}\right)$.

ДокАЗАТЕЛЬСТво. Напомним, что $k$-формула $\tilde{\varphi}_{0}$ не содержит никаких $v$, следовательно, формула $\Phi_{0}(\bar{y})$ утверждает, что существует $x$, при котором формула $\varphi(x, \bar{y}, \bar{w})$ истинна в некоторых точках $\bar{w}$, и что все элементы $\bar{f}_{0}$ и $\bar{e}_{0}$ можно окружить такими точками. Но если существует $x$, при котором формула $\varphi$ истинна во всех точках, то истинно $\Phi_{0}(\bar{y})$ и, следовательно, $\Phi(\bar{y})$.

СЛЕДСТВИЕ 10. Существует константа $M$ такая, что для любого $\bar{y}_{0}$ из истинности

$$
\left(\forall \bar{w}_{1}, \ldots, \bar{w}_{M} \in P\right)(\exists \bar{x})\left(\bigwedge_{i=1}^{M} \varphi\left(x, \bar{y}_{0}, \bar{w}_{i}\right)\right)
$$

следует истинность $\Phi_{0}\left(\bar{y}_{0}\right)$ и, следовательно, $\Phi\left(\bar{y}_{0}\right)$.

ДокАЗАТЕЛЬСтво. Напомним, что длина набора $\bar{u}$ в формуле $\tilde{\varphi}_{0}(x, \bar{y}, \bar{u})$ равна $Q$ ( $\tilde{\varphi}_{0}$ не содержит $\left.v\right)$. Пусть

$$
M=\left(\left|\bar{f}_{K}\right|+\left|\bar{e}_{K}\right|+\cdots+\left|\bar{f}_{1}\right|+\left|\bar{e}_{1}\right|+Q\right)^{N} .
$$

В этой формуле основанием степени является количество элементов множества $G_{0} \cup \operatorname{Set}(\bar{u})$ из формулы $\tilde{\varphi}_{0}^{\prime}(x, \bar{y}, \bar{u})$. Константа $M$, следовательно, является количеством всевозможных наборов длины $N$, которые можно составить из этого множества. Поэтому из истинности

$$
\left(\forall \bar{w}_{1}, \ldots, \bar{w}_{M} \in P\right)(\exists \bar{x})\left(\bigwedge_{i=1}^{M} \varphi\left(x, \bar{y}_{0}, \bar{w}_{i}\right)\right)
$$

следует истинность

$$
(\exists x) \tilde{\varphi}_{0}^{\prime}(x, \bar{y}, \bar{u})
$$

для любого $\bar{u} \in I$, а значит, и истинность $\Phi_{0}\left(\bar{y}_{0}\right)$. 
СлЕДСТВИЕ 11. Если система $\mathfrak{A}$ является әффективно I-сводимой, то константа $M$ находится по формуле $\varphi$ әффективно.

Теперь докажем утверждение в обратную сторону.

Лемма 13. Если $(\mathfrak{A}, I) \models \Phi\left(\bar{y}_{0}\right)$, mo

$$
(\mathfrak{A}, I) \models(\exists x)(\forall \bar{w} \in P) \varphi\left(x, \bar{y}_{0}, \bar{w}\right) .
$$

ДоКАЗАТЕЛЬСТВО. Пусть истинно $\Phi\left(\bar{y}_{0}\right)$. Пусть $i$ - наибольший номер, при котором истинно $\Phi_{i}\left(\bar{y}_{0}\right)$, и $\bar{f}_{i} \in I, \bar{e}_{i} \in I, x_{0}, \bar{a} \in I, \bar{b} \in I$ - элементы, на которых истинна $\Phi_{i}^{\prime}$. Поскольку между соседними элементами $\bar{f}_{i}$ находится не меньше $R+2$ разных элементов $\bar{e}_{i}$, а количество серий в формуле $\varphi_{i}^{\prime}$ не превосходит $R+1$, то хотя бы два из них покрываются одной и той же серией $\bar{a}$, т. е. в любом $\bar{f}_{i}$-промежутке есть элементы, попадаюшие в какую-то неточечную серию $\bar{a}$.

Рассмотрим произвольное конечное множество $S \subseteq I$. Покажем, что сушествует $x_{S}$ такой, что для любого $\bar{s} \in S$ имеет место $\varphi\left(x_{S}, \bar{y}_{0}, \bar{s}\right)$.

Предположим, что для некоторого $s \in S$ имеет место $s \notin[\bar{a}]_{\tilde{\varphi}_{i}}$. Но это означает, что в том же $\bar{f}_{i}$-промежутке, что и $s$, есть крайний элемент какой-то неточечной серии из $\bar{a}$. Переместим элементы $\bar{a}$ и $\bar{b}$ внутри этого $\bar{f}_{i}$-промежутка без изменения их положения относительно друг друга так, чтобы все элементы $S$ из этого $\bar{f}_{i}$-промежутка (а их, конечно, много) оказались бы внутри нового $\left[\bar{a}^{\prime}\right]_{\tilde{\varphi}_{i}}$.

Проделав эту процедуру несколько раз, мы добьемся того, что $S \subseteq\left[\bar{a}^{\prime}\right] \tilde{\varphi}_{i}$. Так как расположение $\bar{a}^{\prime}$ и $\bar{b}^{\prime}$ относительно $\bar{f}_{i}$ и друг друга не изменилось, то согласно лемме 11 сушествует такое $x_{0}^{\prime}$, что для любого $\bar{s} \in S$ выполняется $\varphi\left(x_{0}^{\prime}, \bar{y}_{0}, \bar{s}\right)$. Возьмем это $x_{0}^{\prime}$ в качестве $x_{S}$. Согласно лемме 5 отсюда следует

$$
(\mathfrak{A}, I) \models(\exists x)(\forall \bar{w} \in P) \varphi\left(x, \bar{y}_{0}, \bar{w}\right) .
$$

Лемма 14. Пусть $M$ - константа из следствия 10; тогда

$$
\begin{aligned}
(\exists x)(\forall \bar{w} & \in P) \varphi(x, \bar{y}, \bar{w}) \\
& \Leftrightarrow\left(\forall \bar{w}_{1}, \ldots, \bar{w}_{M} \in P\right)(\exists x) \bigwedge_{i=1}^{M} \varphi\left(x, \bar{y}, \bar{w}_{i}\right) \\
& \Leftrightarrow \Phi_{0}(\bar{y}) \Leftrightarrow \Phi(\bar{y}) .
\end{aligned}
$$

ДоКАЗАТЕЛЬСТВО. Из

$$
(\mathfrak{A}, I) \models(\exists x)(\forall \bar{w} \in P) \varphi\left(x, \bar{y}_{0}, \bar{w}\right)
$$

следует

$$
(\mathfrak{A}, I) \models\left(\forall \bar{w}_{1}, \ldots, \bar{w}_{M} \in P\right)(\exists x) \bigwedge_{i=1}^{M} \varphi\left(x, \bar{y}_{0}, \bar{w}_{i}\right),
$$

отсюда вытекает согласно следствию 10, что

$$
(\mathfrak{A}, I) \models \Phi_{0}\left(\bar{y}_{0}\right), \quad(\mathfrak{A}, I) \models \Phi\left(\bar{y}_{0}\right),
$$

а из последнего по лемме 13 получаем

$$
(\mathfrak{A}, I) \models(\exists x)(\forall \bar{w} \in P) \varphi\left(x, \bar{y}_{0}, \bar{w}\right) .
$$


Tеорема 3. Во всякой малой $I$-сводимой системе $\mathfrak{A}(I \subseteq A)$ для каждой формулы $\varphi(x, \bar{y}, \bar{w})$ сигнатуры $\Sigma$ существует константа $M$ такая, ито в системе $(\mathfrak{A}, I)$

$$
(\exists x)(\forall \bar{w} \in P) \varphi(x, \bar{y}, \bar{w}) \Leftrightarrow\left(\forall \bar{w}_{1}, \ldots, \bar{w}_{M} \in P\right)(\exists x) \bigwedge_{i=1}^{M} \varphi\left(x, \bar{y}, \bar{w}_{i}\right) .
$$

Tеорема 4. Во всякой малой I-сводимой системе $\mathfrak{A}$ для каждой $P$-ограниченной формуль $\varphi(x, \bar{y})$ существует $P$-ограниченная формула $\varphi^{\prime}(\bar{y})$ такая, что в системе $(\mathfrak{A}, I)$

$$
(\exists x) \varphi(x, \bar{y}) \Leftrightarrow \varphi^{\prime}(\bar{y}) .
$$

ДОКАЗАТЕЛЬСТВО следует из леммы 4 и теоремы 3.

ТЕОРема 5. Во всякой малой I-сводимой системе $\mathfrak{A}$ для каждой формуль $\varphi(\bar{y})$ существует Р-ограниченная формула $\varphi^{\prime}(\bar{y})$ такая, что в системе $(\mathfrak{A}, I)$

$$
\varphi^{\prime}(\bar{y}) \Leftrightarrow \varphi(\bar{y}) .
$$

ДокАЗАТЕльСТво. Многократно применяем предыдущую теорему.

СлЕДСТвИЕ 12. Если система $\mathfrak{A}$ әффективно I-сводима, то формула $\varphi^{\prime}$ строится по ч эффективно.

ТЕорема 6. Каждая малая I-сводимая алгебраическая система является тотально I-сводимой.

ДокАЗАТЕЛЬСТво. Для произвольной формулы $\varphi$ в сигнатуре $(\Sigma, P)$ по теореме 5 строим эквивалентную $P$-ограниченную формулу $\varphi^{\prime}$, а для $\varphi^{\prime}$ по определению имеет место $I$-сведение. Следовательно, оно имеет место и для $\varphi$.

СлЕДСТВИЕ 13. Если система является әффективно I-сводимой, то тотальное I-сведение тоэсе является эффективным.

ТЕОРема 7. Если тип неразличимой последовательности I в малой эффективно I-сводимой системе $\mathfrak{A}$ является рекурсивным и система $\mathfrak{A}$ является малой, то тип I в системе (A,I) тоже рекурсивен (в частности, теория системы $(\mathfrak{A}, I)$ разрешима).

ДоКАЗАТЕЛЬСтво. Рассмотрим произвольную формулу $\varphi(\bar{y})$ в сигнатуре $(\Sigma, P)$. Нужно установить истинность $\varphi(\bar{y})$, когда $\bar{y}$ - возрастающая последовательность элементов $I$. Согласно теореме 5 и следствию 12 по формуле $\varphi(\bar{y})$ можно эффективно построить $P$-ограниченную формулу $\varphi^{\prime}(\bar{y})$, эквивалентную $\varphi(\bar{y})$. Формула $\varphi^{\prime}(\bar{y})$ имеет вид

$$
\left(\mathrm{Q}_{1} x_{1} \in P\right) \ldots\left(\mathrm{Q}_{n} x_{n} \in P\right) \varphi^{\prime \prime}(\bar{x}, \bar{y}),
$$

где $\varphi^{\prime \prime}(\bar{x}, \bar{y})$ является формулой в сигнатуре $\Sigma$, a $Q_{1}, \ldots, Q_{n}$ - какие-то кванторные символы. Поскольку тип неразличимой последовательности $I$ в системе $\mathfrak{A}$ рекурсивен, то по $\varphi^{\prime \prime}(\bar{x}, \bar{y})$ эффективно строится порядковая формула $\psi(\bar{x}, \bar{y})$, которая эквивалентна на $I$ формуле $\varphi^{\prime \prime}(\bar{x}, \bar{y})$. А так как теория плотного линейного порядка разрешима, то истинность формулы

$$
\left(\mathrm{Q}_{1} x_{1} \in P\right) \ldots\left(\mathrm{Q}_{n} x_{n} \in P\right) \psi(\bar{x}, \bar{y}),
$$

когда $\bar{y}$ образуют возрастающую последовательность, устанавливается алгоритмически. 
Теорема 8. Если теория $T$ имеет I-сводимую модель, то в теории $T$ выполняется трансляционная теорема.

ДокАЗАТЕЛЬСтво следует из теоремы 6 и [7, теорема 2.6].

Автор выражает благодарность М. А. Тайцлину за обсуждение статьи и ценные советы.

\section{Список литературы}

1. Baldwin J., Benedikt M. Stability theory, permutations of indiscernibles, and embedded finite models // Trans. Amer. Math. Soc. 2000. V. 352. № 11. P. 4937-4969.

2. Belegradek O.V., Stolboushkin A.P., Taitslin M.A. Extended order-generic queries // Annals of Pure and Applied Logic. 1999. V. 97. P. 85-125.

3. Benedikt M., Dong G., Libkin L., Wong L. Relational expressive power of constraint query languages // Proc. 15th ACM Symp. on Principles of Database Systems, 1996. P. $5-16$.

4. Булос Дж., Джефффри Р. Вычислимость и логика. М.: Мир, 1994.

5. Chang C. C., Keisler H. J. Model Theory. Amsterdam: North Holland, 1990.

6. Shelah $S$. Classification theory and the number of non-isomorphic models. Amsterdam: North Holland, 1990.

7. Taitslin M. A. A general condition for collapse results // Annals of Pure and Applied Logic. 2001. V. 113. P. 323-330.

8. Тайцлин $M . A$. Трансляционные результаты в теории баз данных // Сложные системы: обработка информации, моделирование и оптимизация. Тверь: Тверской гос. ун-т, 2002. C. $5-23$.

9. Тайцлин $M$. А. Ограниченные псевдоконечная однородность и изолированность // Вестн. Тверского гос. ун-та. Сер. "Прикладная математика". 2003. Т. 2. № 1. С. 5-15.

Тверской государственный университет

E-mail: Sergey.Dudakov@tversu.ru
Поступило в редакцию 18.02 .2003 